\title{
Bi-modal Gödel logic over [0,1]-valued Kripke frames
}

\author{
Xavier Caicedo * $\quad$ Ricardo Oscar Rodríguez $^{\dagger}$
}

\begin{abstract}
We consider the Gödel bi-modal logic determined by fuzzy Kripke models where both the propositions and the accessibility relation are infinitely valued over the standard Gödel algebra $[0,1]$ and prove strong completeness of Fischer Servi intuitionistic modal logic IK plus the prelinearity axiom with respect to this semantics. We axiomatize also the bi-modal analogues of $T, S 4$, and $S 5$ obtained by restricting to models over frames satisfying the $[0,1]$-valued versions of the structural properties which characterize these logics. As application of the completeness theorems we obtain a representation theorem for bi-modal Gödel algebras.
\end{abstract}

In a previous paper [6], we have considered a semantics for Gödel modal logic based on fuzzy Kripke models where both the propositions and the accessibility relation take values in the standard Gödel algebra $[0,1]$, we call these Gödel-Kripke models, and we have provided strongly complete axiomatizations for the uni-modal fragments of this logic with respect to validity and semantic entailment from countable theories. The systems $\mathcal{G}_{\square}$ and $\mathcal{G}_{\diamond}$ axiomatizing the $\square$-fragment and the $\diamond$-fragment, respectively, are obtained by adding to Gödel-Dummet propositional calculus the following axiom schemes and inference rules:

$$
\begin{aligned}
\mathcal{G}_{\square}: & \square(\varphi \rightarrow \psi) \rightarrow(\square \varphi \rightarrow \square \psi) \\
& \neg \neg \square \varphi \rightarrow \square \neg \neg \varphi \\
& \text { From } \varphi, \text { infer } \square \varphi
\end{aligned}
$$

$$
\begin{aligned}
\mathcal{G}_{\diamond:} & \diamond(\varphi \vee \psi) \rightarrow(\diamond \varphi \vee \diamond \psi) \\
& \diamond \neg \neg \varphi \rightarrow \neg \neg \diamond \varphi \\
& \neg \diamond \perp \\
& \text { From } \varphi \rightarrow \psi, \text { infer } \diamond \varphi \rightarrow \diamond \psi
\end{aligned}
$$

\footnotetext{
*Departamento de Matemáticas, Universidad de los Andes, Bogotá, Colombia; xcaicedo@uniandes.edu.co

${ }^{\dagger}$ Departamento de Computación, Fac. Ciencias Exactas y Naturales, Universidad de Buenos Aires, 1428 Buenos Aires, Argentina; ricardo@dc.uba.ar
} 
These logics diverge substantially in their model theoretic properties. Thus, $\mathcal{G}_{\square}$ does not have the finite model property while $\mathcal{G}_{\diamond}$ does, and the first logic is characterized by models with $\{0,1\}$-valued accessibility relation (accessibility-crisp models) while the second does not. Similar results were obtained for the uni-modal Gödel analogues of the classical modal logics $T$ and $S 4$ determined by Gödel-Kripke models over frames satisfying, respectively, the [0,1]-valued version of reflexivity, or reflexivity and transitivity. The axiomatization of the uni-modal Gödel analogues of $S 5$ remains open.

It is the main purpose of this paper to show that the full bi-modal logic based in Gödel-Kripke models is axiomatized by the system $\mathcal{G}_{\square \diamond}$ which results of adding to the union of $\mathcal{G}_{\square}$ and $\mathcal{G}_{\diamond}$ Fischer-Servi's connecting axioms [14]:

$$
\begin{aligned}
& \diamond(\varphi \rightarrow \psi) \rightarrow(\square \varphi \rightarrow \diamond \psi) \\
& (\diamond \varphi \rightarrow \square \psi) \rightarrow \square(\varphi \rightarrow \psi),
\end{aligned}
$$

and to extend this completeness result to the bi-modal Gödel analogues of classical $T, S 4$, and $S 5$.

The many valued Kripke interpretation of bi-modal logic utilized in this paper was proposed originally by Fitting [15], [16] with a complete Heyting algebra as algebra of truth values, and he gave a complete axiomatization assuming the algebra was finite and the language had constants for all the truth values. In 20] and 21] transformations and characterization of frame properties for these models are given in the general case. Bou, Esteva, and Godo [5] have proposed to utilize this kind of interpretation for general algebras in the study of fuzzy modal logics.

Our method of proof do not seem to extend easily, however, to algebras distinct from the Gödel algebra $[0,1]$ and we do not know any other completeness result for this type of semantics for a fixed algebra $H$, except Fitting's quoted above and Metcalfe \& Olivetti completeness of a natural deduction system for the $\square$-fragment of our Gödel-modal logic [22].

$\mathcal{G}_{\square \diamond}$ may be shown deductively equivalent to the system $I K$ introduced by Fischer-Servi [14 as the natural intuitionistic counterpart of classical modal logic, plus the prelinearity axiom: $(\varphi \rightarrow \psi) \vee(\psi \rightarrow \varphi)$. Similarly, the Gödel analogue of bi-modal $S 5$ results equivalent to the system MIPC of Prior [26] plus prelinearity.

$I K$ and its extensions have been extensively studied, either by means of classical Kripke models for intuitionism equipped with extra relations commuting with the order to interpret the modal operators. ([28], [24], [25], [10], 29], [30, [17], 7], 99]), or by means of algebraic interpretations, specially in the case of $M I P C$, known to be complete for values in monadic 
Heyting algebras (44, 24], 13], 11, 2]) A major result is that under these semantics $I K$ and $M I P C$ enjoy the finite model property.

Clearly, $\mathcal{G}_{\square \diamond}$ and its modal extensions inherit these semantics by asking the multirelational Kripke frames to be linearly ordered or the algebras to be Gödel algebras, but these alternative interpretations do not have the standard character of Gödel-Kripke semantics relevant to fuzzy logic, nor seem our results reducible to their properties. For example, the formula $\square \neg \neg \theta \rightarrow \neg \neg \square \theta$ has finite counter-models in them but not in Gödel-Kripke semantics. We discuss briefly at the end of the paper an embedding of our semantics into algebraic semantics and utilize our completeness theorem to show a representation theorem for countable bi-modal Gödel algebras.

\section{Gödel Kripke models}

The language $\mathcal{L}_{\square \diamond}(V a r)$ of propositional bi-modal logic is built from a set Var of propositional variables, connectives symbols $\vee, \wedge, \rightarrow, \perp$, and the modal operators symbols $\square$ and $\diamond$. Other connectives are defined as usual: $\top:=\varphi \rightarrow \varphi, \neg \varphi:=\varphi \rightarrow \perp, \varphi \longleftrightarrow \psi:=(\varphi \rightarrow \psi) \wedge(\psi \rightarrow \varphi)$. We will write $\mathcal{L}_{\square \diamond}$ if the set $\operatorname{Var}$ is understood.

Recall that a linear Heyting algebra, or Gödel algebra in the fuzzy literature, is a Heyting algebra satisfying the identity $(x \Rightarrow y) \curlyvee(y \Rightarrow x)=1$. The variety of these algebras is generated by the standard Gödel algebra [0,1], the ordered interval with its unique Heyting algebra structure. Let the symbols $\cdot, \Rightarrow, \curlyvee$, and denote, respectively, the meet, residuum (implication), and join operations of $[0,1]$ 1

Definition 1.1 A Gödel-Kripke model (GK-model) will be a structure $M=\langle W, S, e\rangle$ where $W$ is a non-empty set of objects that we call worlds of $M$, and $S: W \times W \rightarrow[0,1], e: W \times \operatorname{Var} \rightarrow[0,1]$ are arbitrary functions. The pair $\langle W, S\rangle$ will be called a $G K$-frame.

The function $e: W \times \operatorname{Var} \rightarrow[0,1]$ associates to each world $x$ a valuation $e(x,-): \operatorname{Var} \rightarrow[0,1]$ which extends to $e(x,-): \mathcal{L}_{\square \diamond}(\operatorname{Var}) \rightarrow[0,1]$ by defining inductively on the construction of the formulas (we utilize the same symbol $e$ to name the extension):

$$
\begin{aligned}
& e(x, \perp):=0 \\
& e(x, \varphi \wedge \psi):=e(x, \varphi) \cdot e(x, \psi)
\end{aligned}
$$

\footnotetext{
${ }^{1}$ The join operation is definable in Gödel algebras as $\left.x \curlyvee y=((x \Rightarrow y) \Rightarrow y)\right) \cdot((y \Rightarrow$ $x) \Rightarrow x)$ )
} 


$$
\begin{aligned}
& e(x, \varphi \vee \psi):=e(x, \varphi) \curlyvee e(x, \psi) \\
& e(x, \varphi \rightarrow \psi):=e(x, \varphi) \Rightarrow e(x, \psi) \\
& e(x, \perp):=0 \\
& e(x, \square \varphi):=\inf _{y \in W}\{S x y \Rightarrow e(y, \varphi)\} \\
& e(x, \diamond \varphi):=\sup _{y \in W}\{S x y \cdot e(y, \varphi)\} .
\end{aligned}
$$

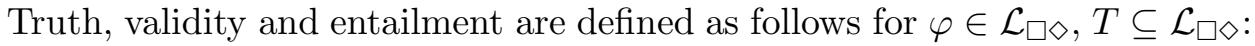

- $\varphi$ is true in $M$ at $x$, written $M \models_{x} \varphi$, if $e(x, \varphi)=1$.

- $\varphi$ is valid in $M$, written $M \models \varphi$, if $M \models_{x} \varphi$ at any world $x$ of $M$.

- $\varphi$ is $G K$-valid, written $\models_{G K} \varphi$, if $M \models \varphi$ for any $G K$-model $M$.

- $T \models_{G K} \varphi$ if and only if for any $G K$-model $M$ and any world $x$ in $M$ : $M \models{ }_{x} \theta$ for all $\theta \in T$ implies $M \models_{x} \varphi$.

It is routine to verify that all axiom schemes corresponding to identities satisfied by $[0,1]$; that is, the laws of Gödel-Dummet logic, are $G K$-valid. In addition

Proposition 1.1 The following schemes are GK-valid:

$$
\begin{array}{ll}
\mathrm{K}_{\square} & \square(\varphi \rightarrow \psi) \rightarrow(\square \varphi \rightarrow \square \psi) \\
\mathrm{K}_{\diamond} & \diamond(\varphi \vee \psi) \rightarrow(\diamond \varphi \vee \diamond \psi) \\
\mathrm{F}_{\diamond} & \neg \diamond \perp \\
\mathrm{FS} 1 & \diamond(\varphi \rightarrow \psi) \rightarrow(\square \varphi \rightarrow \diamond \psi) \\
\text { FS2 } & (\diamond \varphi \rightarrow \square \psi) \rightarrow \square(\varphi \rightarrow \psi) .
\end{array}
$$

Proof: Let $M=\langle W, S, e\rangle$ be a $G K$-model. $\left(\mathrm{K}_{\square}\right)$ By definition and properties of the residuum, $e(x, \square(\varphi \rightarrow \psi)) \cdot e(x, \square \varphi) \leq(S x y \Rightarrow(e(y, \varphi) \Rightarrow$ $e(y, \psi)) \cdot(S x y \Rightarrow e(y, \varphi)) \leq(S x y \Rightarrow e(y, \psi))$ for any $y \in W$. Taking the meet over $y$ in the last expression: $e(x, \square(\varphi \rightarrow \psi)) \cdot e(x, \square \varphi) \leq e(x, \square \psi)$, hence $e(x, \square(\varphi \rightarrow \psi)) \leq e(x, \square \varphi \rightarrow \square \psi)$. $\left(\mathrm{K}_{\diamond}\right)$ By distributivity and properties of the join: $e(\diamond(\varphi \vee \psi))=\sup _{y}\{S x y \cdot(e(y, \varphi) \curlyvee e(y, \psi))\}=$ $\sup _{y}\{S x y \cdot e(y, \varphi)\} \curlyvee \sup _{y}\{S x y \cdot e(y, \psi)\} \cdot\left(\mathbf{F}_{\diamond}\right) e(x, \diamond \perp)=\sup _{y}\{S x y \cdot 0\}=0$. $(\mathrm{FS1}) \operatorname{Sxy} \cdot e(x, \square \varphi) \cdot e(y, \varphi \rightarrow \psi) \leq S x y \cdot(S x y \Rightarrow e(y, \varphi)) \cdot(e(y, \varphi) \Rightarrow e(y, \psi))$ $\leq S x y \cdot e(y, \psi)) \leq e(x, \diamond \psi)$. Therefore, $S x y \cdot e(y, \varphi \rightarrow \psi) \leq(e(x, \square \varphi) \Rightarrow$ $e(x, \diamond \psi))$, and taking the join over $y$ in the left hand side, we have $e(x, \diamond(\varphi \rightarrow \psi)) \leq e(x, \square \varphi \rightarrow \diamond \psi)$. (FS2) $e(x, \diamond \varphi \rightarrow \square \psi) \leq[S x y \cdot e(y, \varphi) \Rightarrow$ $(S x y \Rightarrow e(y, \psi))]=[S x y \cdot e(y, \varphi) \Rightarrow e(y, \psi)]=(S x y \Rightarrow(e, y \rightarrow \psi))$.

Remark. Changing the algebra $[0,1]$ to a complete Heyting algebra $H$ in the above definitions we have Kripke models valued in a $H(H K$ models) and the corresponding notion of $H K$-validity. Then all laws of the 
intermediate logic determined by $H$ are $H K$-valid, and also Proposition 1.1 holds.

\section{A deductive calculus}

Let $\mathcal{G}$ be some axiomatic version of Gödel-Dummet propositional calculus; that is, Heyting calculus plus the prelinearity axiom $(\varphi \rightarrow \psi) \rightarrow(\psi \rightarrow$ $\varphi$ ), and let $\vdash_{\mathcal{G}}$ denote deduction in this logic. Let $\mathcal{L}(X)$ denote the set of formulas built by means of the connectives $\wedge, \rightarrow$, and $\perp$, from a given set $X$. For simplicity, the extension of a valuation $v: X \rightarrow[0,1]$ to $\mathcal{L}(X)$ according to the Heyting interpretation of the connectives will be denoted also $v$.It is well known that this system is complete for validity with respect to these valuations and the distinguished value 1 . We will utilize the fact that it is actually sound and complete in the following strong way (see [6]):

Proposition 2.1 i) If $T \cup\{\varphi\} \subseteq \mathcal{L}(X)$, then $T \vdash_{\mathcal{G}} \varphi$ implies $\inf v(T) \leq v(\varphi)$ for any valuation $v: X \rightarrow[0,1]$. ii) If $T$ is countable, and $T \nvdash_{\mathcal{G}} \varphi_{i_{1}} \vee . . \vee \varphi_{i_{1}}$ for each finite subset of a countable family $\left\{\varphi_{i}\right\}_{i}$ there is a valuation $v: L \rightarrow$ $[0,1]$ such that $v(\theta)=1$ for all $\alpha \in T$ and $v\left(\varphi_{i}\right)<1$ for all $i$.

For an example that completeness for [0,1]-valued entailment can not be extended to uncountable theories see Section 3 in [6] and also Proposition 3.1 in this paper.

Definition 2.1 $\mathcal{G}_{\square \diamond}$ is the deductive calculus obtained by adding to $\mathcal{G}$ the schemes $K_{\square}, K_{\diamond}, F_{\diamond}, F S 1$, FS2 of Proposition 1.1 and the inference rules:

$\mathrm{NR}_{\square} \quad$ From $\varphi$ infer $\square \varphi$

$\mathrm{RN}_{\diamond} \quad$ From $\varphi \rightarrow \psi$ infer $\diamond \varphi \rightarrow \diamond \psi$.

Proofs with assumptions are allowed with the restriction that $\mathrm{NR}_{\square}$ and $\mathrm{RN}_{\diamond}$ may be applied only when the premise is a theorem. Let $\vdash_{\mathcal{G}_{\square \diamond}}$ denote deduction in this system.

The restriction on the application of the rules allows the following convenient reduction (see [6]).

Lemma 2.1 Let $T h \mathcal{G}_{\square \diamond}$ be the set of theorems of $\mathcal{G}_{\square \diamond}$ with no assumptions, then for any theory $T$ and formula $\varphi$ in $\mathcal{L}_{\square \diamond}: T \vdash_{\mathcal{G}_{\square \diamond}} \varphi$ if and only if $T \cup T h \mathcal{G}_{\square \diamond} \vdash_{\mathcal{G}} \varphi$.

and the Deduction Theorem:. 
Lemma 2.2 $T, \psi \vdash_{\mathcal{G}_{\square}} \varphi$ implies $T \vdash_{\mathcal{G}_{\square \triangleright}} \psi \rightarrow \varphi$.

The following are theorems of $\mathcal{G}_{\square \diamond}$. The first one is given as an axiom in Fitting [15, the next two show that $\mathcal{G}_{\square \diamond}$ is just the union of $\mathcal{G}_{\square}, \mathcal{G}_{\diamond}$ plus the Fischer Servi axioms, and the last one will be useful in our completeness proof.

T1. $\neg \diamond \theta \longleftrightarrow \square \neg \theta$

T2. $\neg \neg \square \theta \rightarrow \square \neg \neg \theta$

T3. $\diamond \neg \neg \varphi \rightarrow \neg \neg \diamond \varphi$

T4. $(\square \varphi \rightarrow \diamond \psi) \vee \square((\varphi \rightarrow \psi) \rightarrow \psi)$

To see this, we write temporarily $\vdash$ for $\vdash_{\mathcal{G}_{\square \diamond}}$, then

(T1) $\neg \diamond \theta \vdash(\diamond \theta \rightarrow \square \perp) \vdash \square(\theta \rightarrow \perp)$ by Heyting calculus and FS2. Similarly, $\diamond \theta \vdash \diamond(\neg \theta \rightarrow \perp) \vdash \square \neg \theta \rightarrow \diamond \perp \vdash \neg \square \neg \theta$ by Heyting calculus, $\mathrm{RN}_{\diamond}$, and FS2; hence, $\square \neg \theta \vdash \neg \diamond \theta$.

(T2) $(\square \varphi \rightarrow \perp) \rightarrow \perp \vdash(\square \varphi \rightarrow \diamond \perp) \rightarrow \perp \vdash \diamond(\varphi \rightarrow \perp) \rightarrow \square \perp \vdash$ $\square((\varphi \rightarrow \perp) \rightarrow \perp)$ by $\mathrm{F}_{\diamond}, \mathrm{FS} 2$, and FS1.

(T3) From FS1, $\vdash \diamond(\neg \varphi \rightarrow \perp) \rightarrow(\square \neg \varphi \rightarrow \diamond \perp)$; that is, $\vdash \diamond(\neg \neg \varphi) \rightarrow$ $(\neg \diamond \varphi \rightarrow \perp)$ by $\mathrm{T} 1$ and $\mathrm{F}_{\diamond}$.

(T4) By prelinearity: $\vdash(\square \varphi \rightarrow \diamond(\varphi \rightarrow \psi)) \vee(\diamond(\varphi \rightarrow \psi) \rightarrow \square \varphi)$, but $\square \varphi \rightarrow \diamond(\varphi \rightarrow \psi) \vdash \square \varphi \rightarrow(\square \varphi \rightarrow \diamond \psi) \vdash \square \varphi \rightarrow \diamond \psi$ by FS1; moreover, $\diamond(\varphi \rightarrow \psi) \rightarrow \square \varphi \vdash \square((\varphi \rightarrow \psi) \rightarrow \varphi) \vdash \square((\varphi \rightarrow \psi) \rightarrow \psi)$ by FS2, Heyting calculus and $\mathrm{RN}_{\square}$.

Theorem 2.1 (Soundness) $T \vdash_{\mathcal{G}_{\square} \diamond} \varphi$ implies $T \models_{G K} \varphi$.

Proof: Clearly, the Modus Ponens rule preserves truth at every world of any $G K$-model $M$. Moreover, $M \models \varphi$ implies $M \models \square \varphi$, trivially, and $M \models \varphi \rightarrow \psi$ implies $M \models \diamond \varphi \rightarrow \diamond \psi$ because if $e(x, \varphi \rightarrow \psi)=1$ for all $x$ then $S x y \cdot e(y, \varphi) \leq S x y \cdot e(y, \psi) \leq e(x, \diamond \psi)$ for all $x, y$, and taking the join in the left, $e(x, \diamond \varphi) \leq e(x, \diamond \psi)$. The rest follows from Proposition 1.1.

It is easy to provide counterexamples to the validity of $\neg \square \neg \theta \rightarrow \diamond \theta$ and $\neg \diamond \neg \theta \rightarrow \square \theta$, thus the modal operators are not interdefinable in $\mathcal{G}_{\square \diamond}$ in the classical manner. In fact, they are not interdefinable in any way. For example, the formula $\square \neg \neg \theta \rightarrow \neg \neg \square \theta$ is not expressible in terms of $\diamond$ alone because the $\diamond$-fragment has the finite model property with respect to the number of worlds while this formula has not finite counterexamples as shown in [6].

Remark. $\mathcal{G}_{\square \diamond}$ may be seen deductively equivalent to Fischer-Servi system $I K$ (cf. [14]) plus the prelinearity axiom, replacing $\mathrm{K}_{\square}$ with the axiom 
$\square(\varphi \wedge \psi) \rightarrow(\square \varphi \wedge \square \psi)$ and the rule and $\mathrm{NR}_{\square}$ with From $\varphi \rightarrow \psi$ infer $\square \varphi \rightarrow \square \psi$. Actually, T1, T2, T3 are theorems of $I K$, and we have that $T \vdash_{I K} \varphi$ implies $T \models_{H K} \varphi$ for any complete Heyting algebra $H$.

\section{Completeness}

To prove strong completeness of $\mathcal{G}_{\square \diamond}$ with respect to entailment from countable theories in Gödel-Kripke semantics, our strategy is to show this for finite theories first, and then utilize a first order compactness argument to lift it to countable theories. To show weak completeness we define for each finite fragment $F \subseteq \mathcal{L}_{\square \diamond}$ (that is, a subset closed under subformulas and containing the formula $\perp$ ) a canonical model.

Denote by $\square \mathcal{L}_{\square \diamond}$ and $\diamond \mathcal{L}_{\square \diamond}$ the sets of formulas in $\mathcal{L}_{\square \diamond}$ starting with $\square$

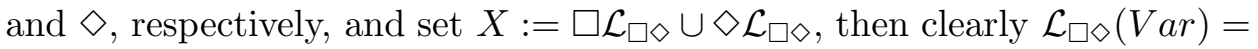
$\mathcal{L}(\operatorname{Var} \cup X)$. Recall that $T h \mathcal{G}_{\square \diamond}$ denotes the set of theorems of $\mathcal{G}_{\square \diamond}$.

The canonical model $M_{F}=\left(W, S^{F}, e^{F}\right)$ is defined as follows.

$W$ : is the set of valuations $v: \operatorname{Var} \cup X \rightarrow[0,1]$ such that $v\left(T h \mathcal{G}_{\square \diamond}\right)=1$ when $T h \mathcal{G}_{\square \diamond}$ is considered as a subset of $\mathcal{L}(\operatorname{Var} \cup X)$.

$S^{F}: S^{F} v w=\inf _{\psi \in F}\{(v(\square \psi) \rightarrow w(\psi)) \cdot(w(\psi) \rightarrow v(\diamond \psi))\}$.

$e^{F}: e^{F}(v, p)=v(p)$ for any $p \in \operatorname{Var}$.

Weak completeness will follow from the following lemma which unfortunately has a rather involved proof.

Lemma 3.1 . $e^{F}(v, \varphi)=v(\varphi)$ for any $\varphi \in F$ and any $v \in W$.

Proof: We prove this by induction in the complexity of the formulas in $F$, now considered a subset of $\mathcal{L}_{\square \diamond}(\operatorname{Var})$. For $\perp$ and the propositional variables in $F$ the equation holds by definition. The only non trivial inductive steps are: $e^{F}(v, \square \varphi)=v(\square \varphi)$ and $e^{F}(v, \diamond \varphi)=v(\diamond \varphi)$ for $\square \varphi, \diamond \varphi \in F$. By the inductive hypothesis we may assume that $e^{F}\left(v^{\prime}, \varphi\right)=v^{\prime}(\varphi)$ for every $v^{\prime} \in W$, thus we must prove

$$
\begin{aligned}
& \left.\inf _{v^{\prime} \in W}\left\{S^{F} v v^{\prime} \Rightarrow v^{\prime}(\varphi)\right\}=v(\square \varphi)\right) \\
& \left.\sup _{v^{\prime} \in W}\left\{S^{F} v v^{\prime} \cdot v^{\prime}(\varphi)\right\}=v(\diamond \varphi)\right)
\end{aligned}
$$


By definition, $S^{F} v v^{\prime} \leq\left(v(\square \varphi) \Rightarrow v^{\prime}(\varphi)\right)$ and $S^{F} v v^{\prime} \leq\left(v^{\prime}(\varphi) \Rightarrow v(\diamond \varphi)\right)$ for any $\varphi \in F$ and $v^{\prime} \in W$; therefore, $v(\square \varphi) \leq\left(S^{F} v v^{\prime} \Rightarrow v^{\prime}(\varphi)\right)$ and $S^{F} v v^{\prime} \cdot v^{\prime}(\varphi) \leq v(\diamond \varphi)$. Taking the meet over $v^{\prime}$ in the first inequality and the join in the second,

$$
v(\square \varphi) \leq \inf _{v^{\prime} \in W}\left\{S^{F} v v^{\prime} \Rightarrow v^{\prime}(\varphi)\right\}, \quad \sup _{v^{\prime} \in W}\left\{S^{F} v v^{\prime} \cdot v^{\prime}(\varphi)\right\} \leq v(\diamond \varphi) .
$$

Hence, if $v(\square \varphi)=1$ and $v(\diamond \varphi)=0$ we obtain (11) and (2), respectively. Therefore, it remains only to prove the next two claims for $\square \varphi, \diamond \varphi \in F$.

Claim 1. If $v(\square \varphi)=\alpha<1$ and $\varepsilon>0$ there exists a valuation $w \in W$ such that $S^{F} v w>w(\varphi)$ and $w(\varphi)<\alpha+\varepsilon$. That is, $\left(S^{F} v w \Rightarrow w(\varphi)\right)<\alpha+\varepsilon$.

Claim 2. If $v(\diamond \varphi)=\alpha>0$ then for any $\varepsilon>0$ there exists $w \in W$ such that $S^{F} v w \cdot w(\varphi) \geq \alpha-\varepsilon$.

Proof of Claim1. Assume $v(\square \varphi)=\alpha<1$ and define (all formulas involved belonging to $\mathcal{L}_{\square \diamond}($ Var $)$ )

$$
\begin{aligned}
\Gamma_{\varphi, v}= & \{\theta: v(\square \theta)>\alpha\} \cup\left\{\theta_{1} \rightarrow \theta_{2}: v\left(\diamond \theta_{1}\right) \leq v\left(\square \theta_{2}\right)\right\} \\
& \cup\left\{\left(\theta_{2} \rightarrow \theta_{1}\right) \rightarrow \theta_{1}: v\left(\diamond \theta_{1}\right)<v\left(\square \theta_{2}\right)\right\} .
\end{aligned}
$$

Then we have $v(\square \xi)>\alpha$ for each $\xi \in \Gamma_{\varphi, v}$. For the first set of formulas by construction. For the second because $v\left(\square\left(\theta_{1} \rightarrow \theta_{2}\right)\right) \geq v\left(\diamond \theta_{1} \rightarrow \square \theta_{2}\right)=1$ by FS2. For the third, because $v\left(\square \theta_{2} \rightarrow \diamond \theta_{1}\right)<1$ and thus $v\left(\square\left(\left(\theta_{2} \rightarrow\right.\right.\right.$ $\left.\left.\left.\theta_{1}\right) \rightarrow \theta_{1}\right)\right)=1$ by $T 4$. This implies that

$$
\Gamma_{\varphi, v} \nvdash_{\mathcal{G}_{\square \diamond}} \varphi .
$$

Otherwise it would exist $\xi_{1}, \ldots, \xi_{k} \in \Gamma_{\varphi, v}$ such that: $\xi_{1}, \ldots, \xi_{k} \vdash_{\mathcal{G}_{\square \diamond}} \varphi$. Hence, $\square \xi_{1}, \ldots, \square \xi_{k} \vdash \vdash_{\square} \square \varphi$, that is $\square \xi_{1}, \ldots, \square \xi_{k}, T h \mathcal{G}_{\square \diamond} \vdash \square \varphi$ by Lemma 2.1 and thus by Proposition 2.1 (i)

$$
\alpha<\inf v\left(\left\{\square \xi_{1}, \ldots, v\left(\square \xi_{k}\right)\right\} \cup T h \mathcal{G}_{\square \diamond)} \leq v(\square \varphi)=\alpha,\right.
$$

a contradiction. Therefore, there exists by Proposition 2.1 (ii) a valuation $u: \operatorname{Var} \cup X \mapsto[0,1]$ such that $u\left(\Gamma_{\varphi, v}^{1} \cup T h \mathcal{G}_{\square \diamond}\right)=1$ and $u(\varphi)<1$. This implies the following relations between $v$ and $u$ that we list for further use (see Figure 1). Given $\theta_{1}, \theta_{2}, \theta_{3}$,

\#1. If $v(\square \theta)>\alpha$ then $u(\theta)=1$ (since then $\theta \in \Gamma_{\varphi, v}^{1}$ ) \#2 If $v\left(\diamond \theta_{1}\right) \leq v\left(\square \theta_{2}\right)$ then $u\left(\theta_{1}\right) \leq u\left(\theta_{2}\right)$ (since then $\theta_{1} \rightarrow \theta_{2} \in \Gamma_{\varphi, v}^{1}$ ) \#3 If $v\left(\diamond \theta_{1}\right)<v\left(\square \theta_{2}\right)$ then $u\left(\theta_{1}\right)=1$ or $u\left(\theta_{1}\right)<u\left(\theta_{2}\right)$ (because then $\left.\left.\left(\theta_{2} \rightarrow \theta_{1}\right) \rightarrow \theta_{1}\right) \in \Gamma_{\varphi, v}^{1}\right)$ 


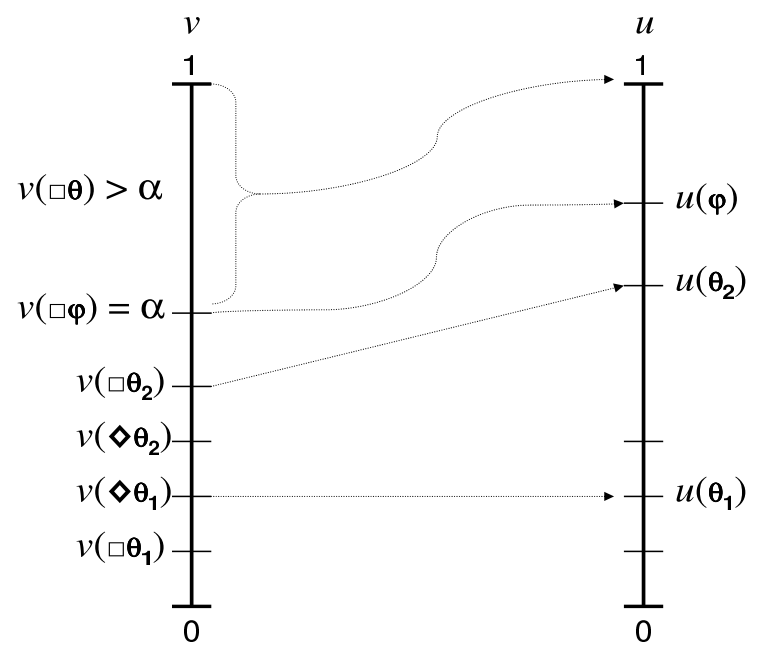

Obs: if $v\left(\diamond \theta_{1}\right)<\alpha$ then $u\left(\theta_{1}\right)<u(\varphi)$

Figure 1: First Translation

\#4. If $u\left(\theta_{2}\right)<u\left(\theta_{1}\right)$ then $v\left(\square \theta_{2}\right)<v\left(\diamond \theta_{1}\right)$ (counter-reciprocal of $\mathbf{2}$ )

\#5. If $v\left(\square \theta_{2}\right)>0$ then $u\left(\theta_{2}\right)>0$ (making $\theta_{1}:=\perp$ in $\mathbf{3}$ because $u(\perp)=$ $v(\diamond \perp)=0)$

\#6. If $u\left(\theta_{2}\right) \leq u\left(\theta_{1}\right)<1$, then $v\left(\square \theta_{2}\right) \leq v\left(\diamond \theta_{1}\right)$ (counter-reciprocal of $\mathbf{3}$ ).

For the next construction we need the finiteness of $F$. Set $B=\{v(\square \theta)$ : $\theta \in F\}$ and for each $b \in B$ define

$$
u_{b}=\min \{u(\theta): \theta \in F, v(\square \theta)=b\},
$$

and then define a strictly descending sequences $b_{0}, b_{1}, \ldots, b_{N}=0$ in $B$ as follows:

$$
\begin{aligned}
& b_{0}=\alpha \\
& b_{i+1}=\max \left\{b<b_{i}: \text { and } u_{b}<u_{b_{i}}\right\}
\end{aligned}
$$

Pick formulas $\varphi_{i} \in F$ such that $b_{i}=v\left(\square \varphi_{i}\right)$ and $u_{b_{i}}=u\left(\varphi_{i}\right)$. By construction the sequence $u_{b_{0}}, u_{b_{1}}, \ldots$ is also strictly descending and $u_{b_{0}}=u_{\alpha} \leq$ $u(\varphi)<1$, thus by finiteness of $F$ the inductive definition ends with some $b_{N}$ (which could be $b_{0}$ in case $u_{\alpha}=0$ ). To check that $b_{N}=u_{b_{N}}=0$, assume 


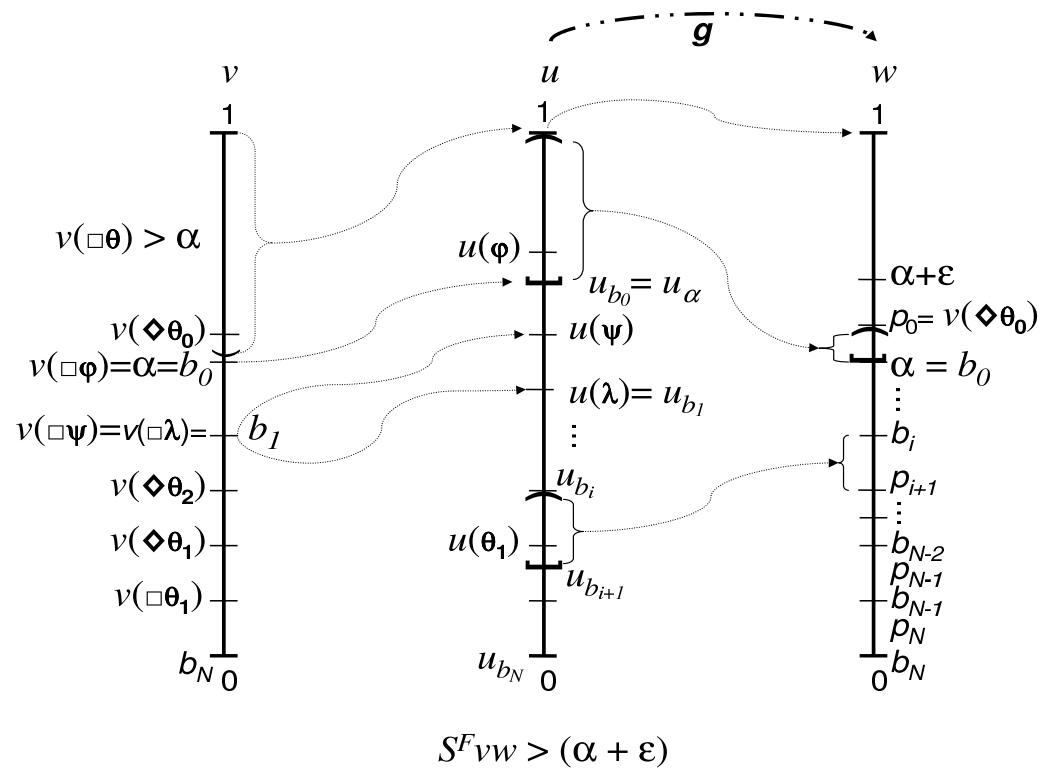

Figure 2: Second Translation

$b_{N}=v\left(\square \varphi_{N}\right)>0$, then $u_{b_{N}}=u\left(\varphi_{N}\right)>0=u(\perp)$ by property \#5 above. Since $v(\square \perp) \leq v\left(\square \varphi_{N}\right)$ then by minimality of $u_{b_{N}}$ we can not have equality, thus $v(\square \perp)<v\left(\square \varphi_{N}\right)$ and there exists $b_{N+1}<b_{N}$, a contradiction. Knowing $v(\square \perp)=b_{N}=0, u_{b_{N}} \leq u(\perp)=0$ by minimality again.

Fix $\varepsilon>0$ such that $\alpha+\varepsilon<1$ and define further (taking $\min \emptyset=1$ )

$$
\begin{aligned}
& p_{0}=(\alpha+\varepsilon) \cdot \min \{v(\diamond \theta): \theta \in F, \alpha<v(\diamond \theta)\} \\
& p_{i}=b_{i+1} \cdot \min \left\{v(\diamond \theta): \theta \in F, b_{i}<v(\diamond \theta)\right\} \text { for } i \geq 1 .
\end{aligned}
$$

We have then $p_{i}>b_{i}$ by finiteness of $F$. Summing up,

$$
\begin{aligned}
& 1>\alpha+\varepsilon \geq p_{0}>b_{0}=\alpha \geq p_{1}>b_{1} \geq \ldots \geq p_{N}>b_{N}=0 . \\
& 1>u_{b_{0}}>u_{b_{1}}>\ldots>u_{b_{N}}=0
\end{aligned}
$$

Now pick an strictly increasing function $g:[0,1] \mapsto[0,1]$ such that (see Figure 2)

$$
\begin{aligned}
& g(1)=1 \\
& g\left[\left[u_{\alpha}, 1\right)\right]=\left[\alpha, p_{0}\right) \\
& g\left[\left[u_{b_{i+1}}, u_{b_{i}}\right)\right]=\left[b_{i+1}, p_{i+1}\right)
\end{aligned}
$$

Then the valuation $w=g \circ u$ satisfies $w\left(T h \mathcal{G}_{\square \diamond}\right)=1$, and so it belongs to $W$. Moreover, for any $\theta \in F$ : 
i) If $u(\theta)=1$ then $w(\theta)=1$ by definition of $w$; hence, $v(\square \theta) \leq w(\theta)$. In addition, $v(\diamond \theta) \geq p_{0}$, otherwise $v(\diamond \theta) \leq \alpha=v\left(\square \varphi_{0}\right)$ which would imply $u(\theta) \leq u\left(\varphi_{0}\right)<1$ by $\# \mathbf{2}$, a contradiction.

ii) If $u(\theta) \in\left[u_{b_{i}}, u_{b_{i-1}}\right)$ or $u(\theta)=\left[u_{b_{0}}, 1\right)$ then $v(\square \theta) \leq w(\theta) \leq v(\diamond \theta)$. To see this notice first that $w(\theta) \in\left[b_{i}, p_{i}\right)$ by definition of $g$. Now, for $i \geq 1$, $b_{i}$ is the maximum $v(\square \psi)$ with $u(\psi)<u_{b_{i-1}}$ therefore $v(\square \theta) \leq b_{i} \leq w(\theta)$. In addition, for $i=0, v(\square \theta) \leq \alpha=b_{0} \leq w(\theta)$ by \#1. Moreover, if $u(\theta)=u_{b_{i}}=u\left(\varphi_{i}\right)$ then $w(\theta)=b_{i}=v\left(\square \varphi_{i}\right) \leq v(\diamond \theta)$ by $\# \mathbf{6}$, and if $u(\theta)>u_{b_{i}}$ then $v(\diamond \theta)>v\left(\square \varphi_{i}\right)=b_{i}$ by \#4; hence, $v(\diamond \theta) \geq p_{i}>w(\theta)$.

From (i,ii), it follows that $\inf _{\theta \in F}\{v(\square \theta) \Rightarrow w(\theta)\}=1$ and $\inf _{\theta \in F}\{w(\theta) \Rightarrow v(\diamond \theta)\} \geq p_{0}$. Hence $S^{F} v w \geq p_{0}$, and $w(\varphi)=g(u(\varphi))<$ $p_{0} \leq \alpha+\varepsilon$.

Proof of Claim 2. Assume $v(\diamond \varphi)=\alpha>0$.

$$
\begin{aligned}
U_{\varphi, v}= & \{\theta: v(\diamond \theta)<\alpha\} \\
& \cup\left\{\vartheta_{2} \rightarrow \vartheta_{1}: v\left(\diamond \vartheta_{1}\right)<v\left(\square \vartheta_{2}\right) \text { and } v\left(\diamond \vartheta_{1}\right)<\alpha\right\} \\
& \cup\left\{\left(\vartheta_{1} \rightarrow \vartheta_{2}\right) \rightarrow \vartheta_{1}: v\left(\diamond \vartheta_{1}\right)=v\left(\square \vartheta_{2}\right) \text { and } v\left(\diamond \vartheta_{1}\right)<\alpha\right\}
\end{aligned}
$$

this set is non-empty because $v(\diamond \perp)=0$, moreover, for any $\xi \in U_{\varphi, v}$ we have $v(\diamond \xi)<\alpha$. For the first set by construction. For the second set of axioms, because $v\left(\diamond\left(\vartheta_{2} \rightarrow \vartheta_{1}\right)\right) \leq v\left(\square \vartheta_{2} \rightarrow \diamond \vartheta_{1}\right)=v\left(\diamond \vartheta_{1}\right)<\alpha$ by FS1. For the third, notice that $\left.v\left(\diamond\left(\left(\vartheta_{1} \rightarrow \vartheta_{2}\right) \rightarrow \vartheta_{1}\right)\right)\right) \leq v\left(\square\left(\vartheta_{1} \rightarrow \vartheta_{2}\right) \rightarrow \diamond \vartheta_{1}\right)$ $\leq v\left(\left(\diamond \vartheta_{1} \rightarrow \square \vartheta_{2}\right) \rightarrow \diamond \vartheta_{1}\right)=v\left(\diamond \vartheta_{1}\right)<\alpha$ by FS1, FS2.

We claim that for any finite $\left\{\xi_{1}, \ldots, \xi_{k}\right\} \subseteq U_{\varphi, v}$ :

$$
\varphi \nvdash_{G \square \diamond} \xi_{1} \vee \ldots \vee \xi_{k}
$$

because, on the contrary, $\diamond \varphi \vdash_{G_{\square}} \diamond\left(\xi_{1} \vee \ldots \vee \xi_{k}\right) \vdash_{G_{\square}} \diamond \xi_{1} \vee \ldots \vee \diamond \xi_{k}$ or

$$
\diamond \varphi, T h \mathcal{G}_{\square \diamond} \vdash \diamond \xi_{1} \vee \ldots \vee \diamond \xi_{k}
$$

and evaluating with $v$ it would give: $\alpha=v(\diamond \varphi) \leq$ $\max \left\{v\left(\diamond \xi_{1}\right), \ldots, v\left(\diamond \xi_{k}\right)\right\}<\alpha$, absurd.

Therefore, there is a valuation $u$ such that $u(\varphi)=u\left(T \mathcal{G}_{\square \diamond}\right)=1$ and $u(\xi)<1$ for each $\xi \in U_{\varphi, v}$, which has the following consequences for any $\theta, \theta_{1}, \theta_{2}$ :

\#\#1. If $v(\diamond \theta)<\alpha$ then $u(\theta)<1$ (because then $\theta \in U_{\varphi, v}$ )

$\# \#$ 2. If $v\left(\diamond \theta_{1}\right)<v\left(\square \theta_{2}\right)$ and $v\left(\diamond \theta_{1}\right)<\alpha$ then $u\left(\theta_{1}\right)<u\left(\theta_{2}\right)$ (because $\left.\theta_{2} \rightarrow \theta_{1} \in U_{\varphi, . v}\right)$

$\# \#$ 3. If $v\left(\diamond \theta_{1}\right) \leq v\left(\square \theta_{2}\right)$ and $v\left(\diamond \theta_{1}\right)<\alpha$ then $u\left(\theta_{1}\right) \leq u\left(\theta_{2}\right)$ (because $\left.\left(\theta_{1} \rightarrow \theta_{2}\right) \rightarrow \theta_{1} \in U_{\varphi, v}\right)$ 
\#\#4 If $u\left(\theta_{2}\right)=0$ then $v\left(\square \theta_{2}\right)=0$ (making $\theta_{1}:=\perp$ in $\mathbf{2}$ and taking counter-reciprocal)

$\# \# \mathbf{5}$. If $v\left(\diamond \theta_{1}\right)=0$ then $u(\theta)=0$ (making $\theta_{2}:=\perp$ in $\mathbf{3}$, because then $v\left(\diamond \theta_{1}\right) \leq v(\square \perp)$ and $\left.v\left(\diamond \theta_{1}\right)<\alpha\right)$.

We perform now a construction dual of the one we did in the proof of Claim 1. Let $C=\{v(\diamond \theta) \leq \alpha: \theta \in F\}$ and define for each $c \in C$

$$
u_{c}=\max \{u(\theta): \theta \in F, v(\diamond \theta)=c\} .
$$

Note that $u_{0}=0$ by \#\# $\mathbf{5}$ above, and $u_{\alpha}=1$ because $u(\varphi)=1$. Define an ascending sequence $0=c_{0}<c_{1}<\ldots$ in $C$ as follows:

$$
\begin{aligned}
& c_{0}=v(\diamond \perp)=0 \\
& c_{1}=\min \left\{c \in C: c>c_{0} \text { and } u_{c}>u_{c_{0}}\right\} \\
& c_{2}=\min \left\{c \in C: c>c_{1} \text { and } u_{c}>u_{c_{1}}\right\}
\end{aligned}
$$

etc.

Choose $\varphi_{i}$ such that $u_{c_{i}}=u\left(\varphi_{i}\right), c_{i}=v\left(\diamond \varphi_{i}\right)$, clearly, $0=u_{c_{0}}<u_{c_{1}}<\ldots$ By finiteness of $F$ the sequence of the $c_{i}$ ends necessarily with $c_{N}=\alpha$, because $c_{i}=v\left(\diamond \varphi_{i}\right)<\alpha$ implies $u_{c_{i}}=u\left(\varphi_{i}\right)<1=u_{\alpha}$ by \#\#1 above and thus the existence of $c_{i+1} \leq \alpha$. This means also that $u_{c_{n}}=1$.

Fix $\varepsilon>0$ such that $\alpha-\varepsilon>c_{N-1}$, and define further (taking $\max \emptyset=0$ )

$$
\begin{aligned}
& q_{N-1}=\max \left\{\alpha-\varepsilon, \max \left\{v(\square \theta): v(\square \theta)<c_{N}\right\}\right\} \\
& q_{i}=\max \left\{c_{i}, \max \left\{v(\square \theta): v(\square \theta)<c_{i+1}\right\}\right\}, \text { for } i<N-1
\end{aligned}
$$

then we have:

$$
\begin{aligned}
& 0=c_{0} \leq q_{0}<c_{1} \leq q_{1}<\ldots . c_{N-1} \leq \alpha-\varepsilon \leq q_{N-1}<c_{N}=\alpha \\
& 0=u_{c_{0}}<u_{c_{1}}<\ldots .<u_{c_{N}}=1
\end{aligned}
$$

Choose $g:[0,1] \rightarrow[0,1]$ to be any strictly increasing function such that

$$
\begin{aligned}
& g(0)=0 \\
& g\left[\left(u_{c_{i}}, u_{c_{i+1}}\right]\right]=\left(q_{i}, c_{i+1}\right] \text { for } i<N-1 \\
& g\left[\left(u_{c_{N-1}}, 1\right)\right]=\left(q_{N-1}, \alpha\right) \\
& g(1)=1
\end{aligned}
$$

Then $g$ is a Heyting homomorphism and the valuation $w=g \circ v$ satisfies $w(\varphi)=w\left(T \mathcal{G}_{\square \diamond}\right)=1$. Moreover, we have:

i) If $v(\diamond \theta) \geq \alpha$ then trivially $(w(\theta) \Rightarrow v(\diamond \theta)) \geq \alpha$. In particular, $(w(\varphi) \Rightarrow$ $v(\diamond \varphi))=(1 \Rightarrow v(\diamond \varphi))=\alpha$. 
ii) If $v(\diamond \theta)<\alpha$ then $w(\theta) \leq v(\diamond \theta)$. To see this consider cases. First: $u(\theta) \in\left(u_{c_{i}}, u_{c_{i+1}}\right)$ for some $i\left(\right.$ recall $u(\theta)<1$ by \#\#1) then $w(\theta) \in\left(q_{i}, c_{i+1}\right]$. As $u(\theta)>u_{c_{i}}$ and $c_{i+1}=v\left(\diamond \varphi_{i+1}\right)$ is the smallest $v(\diamond \psi)$ with $u(\psi)>u_{c_{i}}$ then $v(\diamond \theta) \geq c_{i+1} \geq w(\theta)$. Second: $u(\theta)=0$ then $w(\theta)=0$ and $v(\square \theta)=0$ by \#\#4

iii) If $v(\square \theta) \geq \alpha$ then $(v(\square \theta) \Rightarrow w(\theta))>\alpha-\varepsilon$, because $v(\square \theta)>c_{N-1}=$ $v\left(\diamond \varphi_{N-1}\right)$ which implies $u(\theta)>u\left(\varphi_{N-1}\right)=u_{c_{N-1}}$ by \#\#2, therefore $w(\theta)>q_{N-1} \geq \alpha-\varepsilon$ by definition.

iv) $v(\square \theta)<\alpha$ then $v(\square \theta) \leq w(\theta)$. To see this notice that $c_{i} \leq v(\square \theta) \leq$ $q_{i}<c_{i+1}$ for some $i$ and consider cases. First: $v(\square \theta)=c_{i}=v\left(\diamond \varphi_{i}\right)$ then, by \#\#3, $u_{c_{i}}=u\left(\varphi_{i}\right) \leq u(\theta)$. Therefore $c_{i} \leq w(\theta)$. That is, $v(\square \theta) \leq w(\theta)$. Second: $c_{i}<v(\square \theta)$ then, by \#\#2, $u_{c_{i}}<u(\theta)$ and by definition $q_{i} \leq w(\theta)$, which shows again $v(\square \theta) \leq w(\theta)$.

From (i,ii), we have $\inf _{\theta \in F}\{w(\theta) \Rightarrow v(\diamond \theta)\}=\alpha$, and from (iii,iv), $\inf _{\theta \in F}\{v(\square \theta) \Rightarrow w(\theta)\} \geq \alpha-\varepsilon$. Hence, $S^{F} v w \geq \alpha-\varepsilon$ and thus $S^{F} v w \cdot w(\varphi)=$ $S^{F} v w \cdot \alpha \geq(\alpha-\varepsilon)$.

Lemma 3.2 (Weak completeness) For any finite theory $T$ and formula

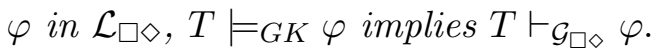

Proof: Assume $T$ is finite and $T \nvdash_{\mathcal{G}_{\square}} \varphi$ then $T, T h \mathcal{G}_{\square \diamond} \nvdash_{\mathcal{G}} \varphi$ by Lemma 2.1 and thus there is by Proposition 2.1 a Gödel valuation $v: \operatorname{Var} \cup X \rightarrow[0,1]$ such that $v(\varphi)<v(T)=v\left(T h \mathcal{G}_{\square \diamond}\right)=1$. Let $F$ be the set of subformulas of formulas in $T \cup\{\varphi\}$ (including $\perp$ ), then $v \in W$. the set of worlds of the canonical model $M_{F}$ and by Lemma 3.1, $e^{F}(v, T)=v(T)=1$ and $e^{F}(v, \varphi)=v(\varphi)<1$, thus $T \nvdash_{G K} \varphi$.

To prove strong completeness we utilize compactness of first order classical logic and the following result of Horn:

Lemma 3.3 ([19, Lemma 3.7) Any countable linear order $(P,<)$ may be embedded in $(\mathbb{Q} \cap[0,1],<)$ preserving all joins and meets existing in $P$.

Theorem 3.1 (Strong completeness) For any countable theory $T$ and

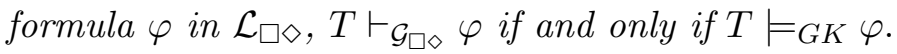

Proof: Assume $T$ is countable and $T \nvdash_{\mathcal{G}_{\square \diamond}} \varphi$. Consider the first order theory $T^{*}$ with two unary relation symbols $W, P$, binary $<$, constant symbols 0,1 , and $c$, function symbols $x \circ y, S(x, y)$, and $f_{\theta}(x)$ for each $\theta \in \mathcal{L}_{\square \diamond}(V)$ where $V$ is the set of propositional variables of $T$, and having for axioms:

$$
\forall x \neg(W x \wedge P x)
$$


$(P,<)$ is a strict linear order with minimum 0 and maximum 1

$\forall x \forall y(W(x) \wedge W(y) \rightarrow P(S(x, y)))$

$\forall x \forall y(P(x) \wedge P(y) \rightarrow(x \leq y \wedge x \circ y=1) \vee(x>y \wedge x \circ y=y))$

$\forall x\left(W(x) \rightarrow f_{\perp}(x)=0\right)$

for each $\theta, \psi \in \mathcal{L}_{\square \diamond}$ :

$\forall x\left(W(x) \rightarrow P\left(f_{\theta}(x)\right)\right)$

$\forall x\left(W(x) \rightarrow f_{\theta \wedge \psi}(x)=\min \left\{f_{\theta}(x), f_{\psi}(x)\right\}\right)$

$\forall x\left(W(x) \rightarrow f_{\theta \rightarrow \psi}(x)=\left(f_{\varphi}(x) \circ f_{\psi}(x)\right)\right.$

$\forall x\left(W(x) \rightarrow f_{\square \theta}(x)=\inf _{y}\left(S(x, y) \circ f_{\theta}(y)\right)\right.$

$\forall x\left(W(x) \rightarrow f_{\diamond \theta}(x)=\sup _{y}\left(\min \left\{S(x, y), f_{\theta}(y)\right\}\right)\right.$

$W(c) \wedge\left(f_{\varphi}(c)<1\right)$

for each $\theta \in T: f_{\theta}(c)=1$

For each finite part $t$ of this theory let $\Sigma_{t}=\left\{\theta: f_{\theta} \in t\right\}$. Since $\Sigma_{t} \cap T \nvdash_{\mathcal{G}_{\square \diamond}} \varphi$ by hypothesis then by weak completeness there is a GKmodel $M_{\Sigma}=\left(W_{\Sigma}, S_{\Sigma}, e_{\Sigma}\right)$ and $a \in W_{\Sigma}$ such that $e_{\Sigma}(a, \theta)=1$ for each $\theta \in \Sigma_{t} \cap T$ and $e_{\Sigma}(a, \varphi)<1$. Therefore the first order structure $\left(W_{\Sigma} \sqcup[0,1], W_{\Sigma},[0,1],<, 0,1, a, \Rightarrow, S_{\Sigma}, f_{\theta}\right)_{\theta \in \mathcal{L}_{\square \diamond}}$, with $f_{\theta}: W_{\Sigma} \rightarrow[0,1]$ defined as $f_{\theta}(x)=e_{\Sigma}(x, \theta)$, is clearly a model of $t$. By compactness of first order logic and the downward Löwenheim theorem $T^{*}$ has a countable model $M^{*}=\left(B, W, P,<, 0,1, a, \circ, S, f_{\theta}\right)_{\theta \in \mathcal{L}_{\square \diamond}}$. Using Horn's lemma [19], $(P,<)$ may be embedded in $(\mathbb{Q} \cap[0,1],<)$ preserving 0,1 , and all suprema and infima existing in $P$; therefore, we may assume without loss of generality that the ranges of the functions $S$ and $f_{\theta}$ are contained in $[0,1]$. Then, it is straightforward to verify that $M=(W, S, e)$, where $e(x, \theta)=f_{\theta}(x)$ for all $x \in W$, is a GK-model such that $M \models_{a} T$, and $M \nvdash_{a} \varphi$, that is, $T \nvdash_{G K} \varphi$. The rest follows from Theorem 2.1.

We can not expect a similar result for uncountable theories by the observation after Proposition 2.1. If we allow non-standard values, for example $H K$-models where $H$ is an adequate ultrapower of $[0,1]$, we may obtain strong completeness for entailment of uncountable theories up to certain cardinality. However,

Proposition 3.1 There is no single linearly ordered Heyting algebra $H$ giving strong completeness with respect to HK models for theories of arbitrary power, even in Gödel-Dummet logic.

Proof: Assume otherwise, then $H$ would be infinite (by the old Gödel argument). Let $\kappa$ be a cardinal greater than $|H|$ and consider the theory $T=\left\{\left(p_{\beta} \rightarrow p_{\alpha}\right) \rightarrow q: \alpha<\beta<\kappa\right\}$, then $T \models_{H K} q$ because $v(T)=1$ 
with $v(q)<1$ would imply $v\left(p_{\beta} \rightarrow p_{\alpha}\right)<1$ and thus $v\left(p_{\alpha}\right)<v\left(p_{\beta}\right)$ for $\alpha<\beta<\kappa$, which is impossible by cardinality considerations. On the other hand, $T \nvdash_{\mathcal{G}_{\square \diamond}} q$, otherwise we would have $\Delta \vdash_{\mathcal{G}_{\square} \diamond} q$ and thus $\Delta \models_{H K} q$, for some finite set $\Delta=\left\{\left(p_{\alpha_{i+1}} \rightarrow p_{\alpha_{i}}\right) \rightarrow q: 1 \leq i<n\right\}$, which is impossible because any valuation $v(q)=h, v\left(p_{\alpha_{i}}\right)=h_{i}$ where $h_{1}<h_{2}<\ldots<h_{n+1}<h<1$ makes $v\left(\left(p_{\alpha_{i+1}} \rightarrow p_{\alpha_{i}}\right) \rightarrow q\right)=1$ for $1 \leq i<n$.

\section{Optimal models, modal axioms}

To extend the completeness theorem to the $[0,1]$-valued analogues of the classical bi-modal systems $T, S 4, S 5$ we introduce a particular kind of GKmodel, their advantage being that the many-valued counterpart of classical structural properties of frames may be characterized in them by the validity of the corresponding classical schemes.

Given a GK-model $M=(W, S, e)$, define a new accessibility relation $S^{+} x y=S_{\square} x y \cdot S_{\diamond} x y$, where $S_{\square} x y=\inf _{\varphi \in \mathcal{L}_{\square \diamond}}\{e(x, \square \varphi) \Rightarrow e(y, \varphi)\}$, and $S_{\diamond} x y=\inf _{\varphi \in \mathcal{L}_{\square \diamond}}\{e(y, \varphi) \Rightarrow e(x, \diamond \varphi)\}$, and call $M$ optimal if $S^{+}=S$.

The following lemma shows that any model is equivalent to an optimal one.

Lemma $4.1\left(W, S^{+}, e\right)$ is optimal and if $e^{+}$is the extension of $e$ in this model then $e^{+}(x, \varphi)=e(x, \varphi)$ for any $\varphi \in \mathcal{L}_{\square \diamond}$.

Proof: The first claim follows from the second which is proven by a straightforward induction on formulas. The only non trivial step is that of the modal connectives. Notice first that $S x y \leq S^{+} x y$, because $e(x, \square \varphi) \leq$ $(S x y \Rightarrow e(y, \varphi))$ and $S x y \cdot e(y, \varphi) \leq e(x, \diamond \varphi)$ for any $\varphi$, thus $S x y \leq$ $(e(x, \square \varphi) \Rightarrow e(y, \varphi)),(e(y, \varphi) \Rightarrow e(x, \diamond \varphi))$. Now, assume $e^{+}(y, \varphi)=e(y, \varphi)$ for all $y$ then by the first observation and the induction hypothesis, $e^{+}(x, \square \varphi)=\inf _{y}\left\{S^{+} x y \Rightarrow e^{+}(y, \varphi)\right\} \leq \inf _{y}\{S x y \Rightarrow e(y, \varphi)\}=e(x, \square \varphi)$. But $S^{+} x y \leq(e(x, \square \varphi) \Rightarrow e(y, \varphi))$ by definition of $S^{+}$and thus $e(x, \square \varphi) \leq$ $\left(S^{+} x y \Rightarrow e(y, \varphi)\right)=\left(S^{+} x y \Rightarrow e^{+}(y, \varphi)\right)$ which yields $e(x, \square \varphi) \leq e^{+}(x, \square \varphi)$. Similarly, by the induction hypothesis and the first observation, $e^{+}(x, \diamond \varphi)=$ $\sup _{y}\left\{S^{+} x y \cdot e(y, \varphi)\right\} \geq \sup _{y}\{S x y \cdot e(y, \varphi)\}=e(x, \diamond \varphi)$, and by definition $S^{+} x y \leq(e(y, \varphi) \Rightarrow e(x, \diamond \varphi))$ and thus $S^{+} x y \cdot e^{+}(y, \varphi)=S^{+} x y \cdot e(y, \varphi) \leq$ $e(x, \diamond \varphi)$ which yields $e^{+}(x, \diamond \varphi) \leq e(x, \diamond \varphi)$.

Call a GK-frame $\mathcal{M}=\langle W, S\rangle$ reflexive if $S x x=1$ for all $x \in W$, transitive if $S x y \cdot S y z \leq S x z$ for all $x, y, z$, and symmetric if $S x y=S y x$ for 
all $x, y \in W$. Let Ref, Trans, and Symm denote, respectively, the classes of GK- models over frames satisfying each one of the above properties. These are the fuzzy versions of the corresponding classical properties of frames characterized by the following pairs of modal axioms:

$$
\begin{array}{lll}
\mathrm{T}_{\square} . \quad \square \varphi \rightarrow \varphi & \mathrm{T}_{\diamond .} \quad \varphi \rightarrow \diamond \varphi & \text { reflexivity } \\
4_{\square} . \quad \square \varphi \rightarrow \square \square \varphi & 4_{\diamond .} \diamond \diamond \varphi \rightarrow \diamond \varphi & \text { transitivity } \\
\mathrm{M}_{1} . \quad \varphi \rightarrow \square \diamond \varphi & \mathrm{M}_{2} . \quad \diamond \square \varphi \rightarrow \varphi & \text { symmetry }
\end{array}
$$

We will see that these axioms characterize also the fuzzy versions in optimal models.

Lemma 4.2 i) $T_{\square}$ and $T_{\diamond}$ are valid in Ref. ii) $4_{\square}$ and $4_{\diamond}$ are valid in Trans. iii) $M_{1}$ and $M_{2}$ are valid in Symm.

Proof: i) In reflexive models, $e(x, \square \varphi) \leq(S x x \Rightarrow e(x, \varphi))=e(x, \varphi)$ and $e(x, \diamond \varphi) \geq S x x \cdot e(x, \varphi)=e(x, \varphi)$ for any $x$. Thus $e(x, \square \varphi \rightarrow \varphi)=1=$ $e(x, \varphi \rightarrow \diamond \varphi)$.

ii) In transitive models $e(x, \square \varphi) \cdot S x y \cdot S y z \leq[(S x z \Rightarrow e(z, \varphi)) \cdot S x z] \leq$ $e(z, \varphi)$ for all $x, y, z$. Hence, $e(x, \square \varphi) \cdot S x y \leq(S y z \Rightarrow e(z, \varphi))$ and thus $e(x, \square \varphi) \cdot S x y \leq e(y, \square \varphi)$; therefore, $e(x, \square \varphi) \leq(S x y \Rightarrow e(y, \square \varphi))$ for all $y$ and thus $e(x, \square \varphi) \leq e(x, \square \square \varphi)$ which yields $4_{\square}$. Also $S x y \cdot S y z \cdot e(z, \varphi)$ $\leq S x z \cdot e(z, \varphi) \leq e(x, \diamond \varphi)$. Hence, $S y z \cdot e(z, \varphi) \leq(S x y \Rightarrow e(x, \diamond \varphi))$ and thus $e(x, \diamond \varphi) \leq(S x y \Rightarrow e(x, \diamond \varphi))$; therefore, $S x y \cdot e(x, \diamond \varphi) \leq e(x, \diamond \varphi))$ for all $y$ and thus $e(x, \diamond \diamond \varphi) \leq e(x, \diamond \varphi)$ which gives $4 \diamond$. iii) In symmetric models, $S x y \cdot e(x, \varphi)=S y x \cdot e(x, \varphi) \leq e(y, \diamond \varphi)$ for all $x, y$, then $e(x, \varphi) \leq$ $(S x y \Rightarrow e(y, \diamond \varphi))$ and thus $e(x, \varphi) \leq e(y, \square \diamond \varphi))$ which is $\mathrm{M}_{1}$. Moreover, $e(y, \square \varphi) \leq(S y x \Rightarrow e(x, \varphi))$, thus $S x y \cdot e(y, \square \varphi)=S y x \cdot e(y, \square \varphi) \leq e(x, \varphi)$ and thus $e(x, \diamond \square \varphi) \leq e(x, \varphi)$ which is $\mathrm{M}_{2}$.

Proposition 4.1 Let $M$ be an optimal GK-model, then i) It is reflexive if and only if it validates the schemes $T_{\square}+T_{\diamond}$. ii) It is transitive if and only if it validates $4_{\square}+4_{\diamond}$. iii) It is symmetric if and only if it validates $M_{1}+M_{2}$.

Proof: i) By optimality, $S x x=\inf _{\varphi}\{e(x, \square \varphi \rightarrow \varphi)\} \cdot \inf _{\varphi}\{e(x, \varphi \rightarrow \diamond \varphi)\}=$ 1 if $\mathrm{T}_{\square}, \mathrm{T}_{\diamond}$ hold in $M$. ii) By definition,

$$
\begin{aligned}
S_{\square} x y \cdot S_{\square} y z & \leq(e(x, \square \square \varphi) \Rightarrow e(y, \square \varphi)) \cdot(e(y, \square \varphi) \Rightarrow e(z, \varphi)) \\
& \leq(e(x, \square \square \varphi) \Rightarrow e(z, \varphi)) \leq(e(x, \square \varphi) \Rightarrow e(z, \varphi)),
\end{aligned}
$$

the last inequality holding by $4 \square$. Similarly

$$
\begin{aligned}
S_{\diamond} x y \cdot S_{\diamond} y z & \leq(e(y, \diamond \varphi) \Rightarrow e(x, \diamond \diamond \varphi)) \cdot(e(z, \varphi) \Rightarrow e(y, \diamond \varphi)) \\
& \leq(e(z, \varphi) \Rightarrow e(x, \diamond \diamond \varphi)) \leq(e(z, \varphi) \Rightarrow e(x, \diamond \varphi)
\end{aligned}
$$


the last inequality holding by $4_{\diamond}$, and taking meet over $\varphi$ in the right we get transitivity. iii) since $S_{\square} x y \leq(e(x, \square \diamond \varphi) \Rightarrow e(y, \diamond \varphi)) \leq(e(x, \varphi) \Rightarrow$ $e(y, \diamond \varphi))$ by $\mathrm{M}_{1}$, then taking meet over $\varphi$, we obtain $S_{\square} x y \leq S_{\diamond} y x$. Similarly, $S_{\diamond} y x \leq(e(x, \square \varphi) \Rightarrow e(y, \diamond \square \varphi)) \leq(e(x, \square \varphi) \Rightarrow e(y, \varphi))$ by $\mathrm{M}_{2}$, and then $S_{\diamond} y x \leq S_{\square} x y$. From this, $S_{\diamond x y}=S_{\square} y x$, and thus $S x y=S y x$.

Remark. The notion of optimality and all the results in this section make sense and hold for $H K$-models, for any complete Heyting algebra $H$.

\section{Gödel analogues of classical bi-modal systems}

Lemma 4.1 in conjunction with Proposition 4.1 implies strong completeness of any combination of the axiom pairs in table 3 with respect to GK-frames satisfying the associated structural properties. In particular, we have for the analogues of the classical modal systems T, S4 and S5:

$$
\begin{array}{ll}
\mathcal{G} T_{\square \diamond}:=\mathcal{G} \square \diamond+T_{\square}+T_{\diamond} \\
\mathcal{G} S 4_{\square \diamond}:=\mathcal{G} T_{\square \diamond}+4_{\square}+4 \diamond \\
\mathcal{G} S 5_{\square \diamond}:=\mathcal{G} S 4_{\square \diamond}+M 1+M 2 .
\end{array}
$$

Theorem 5.1 i) $\mathcal{G} T_{\square \diamond}$ is strongly complete for $\models_{\text {Ref }}$. ii) $\mathcal{G} S 4_{\square \diamond}$ is strongly complete for $\models$ RefกTrans . iii) $\mathcal{G} S 5_{\square \diamond}$ is strongly complete for

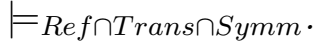

Proof: i) If $T \models_{\operatorname{Ref}} \varphi$ then $T \models_{\text {Optimaln } \operatorname{Ref}} \varphi$, thus $T+\left\{T_{\square}, T_{\diamond}\right\} \models_{\text {Optimal }} \varphi$ by Proposition 4.1, and $T+\left\{T_{\square}, T_{\diamond}\right\} \models_{\mathrm{GK}} \varphi$ by Lemma 4.1,Therefore, $T+\left\{T_{\square}, T_{\diamond}\right\} \vdash_{\mathcal{G}_{\square \diamond}} \varphi$, which implies $T \vdash_{\mathcal{G} T_{\square \diamond}} \varphi$. Here, we have used $\{\ldots\}$ to denote the set of all instances of the schemes within the brackets. The proofs of (ii) and (iii) are similar.

After some calculation $\mathcal{G} S 5 \square \diamond$ may be seen deductively equivalent to Prior's and Bull system MIPC [26], [4] plus the prelinearity axiom $(\varphi \rightarrow$ $\psi) \vee(\psi \rightarrow \varphi)$. This system presents some interesting features with respect to its Gödel-Kripke semantics, given by GK-frames $(W, S)$ where $S$ is a fuzzy equivalence relation. Although the uni-modal fragments of $\mathcal{G} T_{\square \diamond}$ and $\mathcal{G} S 4_{\square \diamond}$ are axiomatizable by the double negation shift axioms and the proper axioms in 3, as shown [6], the intrinsic axiomatization of the uni-modal fragments of $\mathcal{G} S 5_{\square} \diamond$ remains open. Moreover, in distinction of the other modal systems here considered, the $\square$-fragment of $\mathcal{G} S 5 \square \diamond$ is not characterized by models with crisp accessibility relation, as the following example illustrates. 
Example The formula $\square(\square \varphi \vee \psi) \rightarrow(\square \varphi \vee \square \psi)$ is not a theorem of $\mathcal{G} S 5_{\square}$ but it is valid in any accessibility-crisp model of $\mathcal{G} S 5_{\square}$. The first claim is granted by the following counter model:

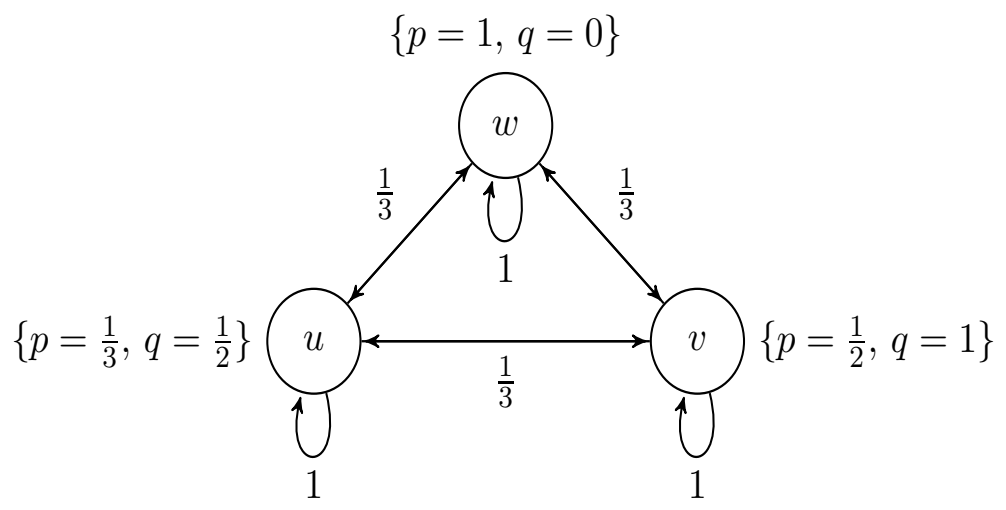

in which the reader may verify that $e\left(u, \square(\square p \vee q)=\frac{1}{2}\right.$ and $e(u, \square p \vee \square q)=\frac{1}{3}$. To prove the second claim notice that if $(W, S, e) \in \operatorname{Re} f \cap$ Trans $\cap S y m m$ has crisp $S$, this defines a classical equivalence relation $\sim$ and thus $e(x, \square \varphi)=$ $\inf _{y}\{S x y \Rightarrow e(y, \varphi)\}=\inf _{y \sim x}\{e(y, \varphi)\}$ for any formula $\varphi$. Therefore, $e(x, \square(\square \varphi \vee \psi))=\inf _{y \sim x}\left\{\inf _{z \sim y} e(z, \varphi) \curlyvee e(y, \varphi)\right\}$, but $\{z: z \sim y\}=\{z:$ $z \sim x\}$ and so $\alpha_{y}=\inf _{z \sim y} e(z, \varphi)$ is independent of $y$ for any $y \sim x$; hence, $e(x, \square(\square \varphi \vee \psi))=\inf _{y \sim x}\{\alpha \curlyvee e(y, \varphi)\}=\alpha \curlyvee \inf _{y \sim x}\{e(y, \varphi)\}=e(x, \square \varphi \vee \square \psi)$ by properties of $[0,1]$.

\section{The algebraic connection}

As an algebrizable deductive logic, $\mathcal{G}_{\square \diamond}$ has a unique algebraic semantics given by the variety of bi-modal Gödel algebras $A=(G, I, K)$ where $G$ is a Gödel algebra and $I$ and $K$ are unary operations in $G$ satisfying the identities:

$$
\begin{array}{ll}
I(a \cdot b)=I a \cdot I b & K(a \curlyvee b)=K a \curlyvee K b \\
I 1=1 & K 0=0 \\
K a \rightarrow I b \leq I(a \rightarrow b) & K(a \rightarrow b) \leq I a \rightarrow K b
\end{array}
$$

This means that $\mathcal{G}_{\square \diamond}$ is complete with respect to valuations $v: \operatorname{Var} \rightarrow A$ in these algebras, when they are extend to $\mathcal{L}_{\square \diamond}$ interpreting $\square$ and $\diamond$ by $I$ and $K$, respectively.

Similarly, $\mathcal{G} T_{\square \diamond}, \mathcal{G} S 4_{\square \diamond}$, and $\mathcal{G} S 5_{\square \diamond}$ have for algebraic semantic the subvarieties of bi-modal Gödel algebras determined by the corresponding pairs of identities in the following table: 


$$
\begin{array}{lll}
I a \leq a & a \leq K a & \text { reflexivity } \\
I a=I I a & K a=K K a & \text { transitivity } \\
a \leq I K a & K I a \leq a & \text { symmetry }
\end{array}
$$

Notice that the algebraic models of $\mathcal{G} S 4_{\square \diamond}$ are just the bi-topological pseudoBoolean algebras of Ono 24] with linear underlying Heyting algebra, and the algebraic models of $\mathcal{G} S 5_{\square} \diamond$ are the the monadic Heyting algebras of Monteiro and Varsavsky [23], utilized later by Bull and Fischer Servi to interpret MIPC, with a Gödel basis. It is proper to call them monadic Gödel algebras.

Example. As we have noticed, there is no finite counter-model for the formula $\square \neg \neg p \rightarrow \neg \neg \square p$ in Gödel-Kripke semantics. However, the algebra $A=(\{0, a, 1\}, I, K)$ where $\{0<a<1\}$ is the three elements Gödel algebra and $I 1=1, I a=I 0=0, K 1=K a=1, K 0=0$ is a bi-modal Gödel algebra (actually a monadic Heyting algebra) providing a finite counterexample to the validity of the formula by means of the valuation $v(p)=a$, as the reader may verify.

We may associate to each Gödel-Kripke frame $\mathcal{F}=(W, S)$ a bi-modal Gödel algebra $[0,1]^{\mathcal{F}}=\left([0,1]^{W}, I^{\mathcal{F}}, K^{\mathcal{F}}\right)$ where $[0,1]^{W}$ is the product Gödel algebra, and for each map $f \in[0,1]^{W}$ :

$$
\begin{aligned}
I^{\mathcal{F}}(f)(w) & =\inf _{w^{\prime} \in W}\left(S w w^{\prime} \Rightarrow f\left(w^{\prime}\right)\right) \\
K^{\mathcal{F}}(f)(w) & =\sup _{w^{\prime} \in W}\left(S w w^{\prime} \cdot f\left(w^{\prime}\right)\right)
\end{aligned}
$$

Theorem 6.1 $[0,1]^{\mathcal{F}}$ is a bi-modal Gödel algebra, and there is a one to one correspondence between Gödel Kripke models over $\mathcal{F}$, and valuations $v: \operatorname{Var} \rightarrow[0,1]^{\mathcal{F}}$ given by the adjunction:

$$
\operatorname{Var} \times W \stackrel{e}{\rightarrow}[0,1] \leftrightarrow \operatorname{Var} \stackrel{v_{e}}{\rightarrow}[0,1]^{W}, \quad v_{e}(p)=e(-, p)
$$

so that for any formula $\varphi, v_{e}(\varphi)=e(-, \varphi)$.

Moreover, the transformation $\mathcal{F} \longmapsto[0,1]^{\mathcal{F}}$ preserves reflexivity, transi-

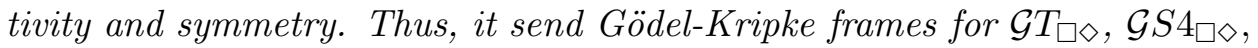
and $\mathcal{G} S 5_{\square \diamond}$ into algebraic models for the same logics.

Proof: The verification of the identities that $I^{\mathcal{F}}, K^{\mathcal{F}}$ must satisfy in each case is routine and the induction in formulas showing $v_{e}(\varphi)(w)=e(w, \varphi)$ is straightforward. 
Call an algebra of the form $[0,1]^{\mathcal{F}}$ a Gödel complex algebra. Going from algebras to GK-models seems more difficult. However, utilizing a refinement of our strong completeness theorem for Gödel-Kripke semantics we may associate to each countable bi-modal Gödel algebra $A$ a GK-frame $\mathcal{F}_{A}$ such that $A$ may be embedded in the associated algebra $[0,1]^{\mathcal{F}_{A}}$, and to each algebraic valuation $\eta$ in $A$ a GK-model over $\mathcal{F}_{A}$ validating the same formulas as $\eta$.

Call a theory $T \subseteq \mathcal{L}_{\square \diamond}$ normal if $T \vdash_{\mathcal{G}_{\square \diamond}} \theta$ implies $T \vdash_{\mathcal{G}_{\square \diamond}} \square \theta$ and $T$ $\vdash_{\mathcal{G}_{\square \diamond}} \theta \rightarrow \rho$ implies $T \vdash_{\mathcal{G}_{\square \diamond}} \diamond \theta \rightarrow \diamond \rho$.

It $T$ is normal, then for each finite fragment $F$ the proof of the Lemma 3.1 goes through for the submodel $M_{F}^{T}=\left(W^{T}, S^{F}, e^{F}\right)$ of the canonical model where $W^{T}=\{v \in W: v(T)=1\}$. Hence, if $\Sigma$ is a finite subset of $T$ such that $\Sigma \nvdash_{\mathcal{G}_{\square \diamond}} \varphi$ there is a canonical model $M_{F}^{T}$ such that $e^{F}(v, \Sigma)=1$ and $e^{F}(v, \varphi)<1$ (take $\left.F \supseteq \Sigma \cup\{\varphi\}\right)$.

Lemma 6.1 If $T$ is a countable normal theory there is GK-model $M_{T}$ such that $T \vdash_{\mathcal{G}_{\square \diamond}} \varphi$ if and only if $M_{T} \models \varphi$.

Proof: From the previous observation, and utilizing a compactness argument as in the proof of Theorem 3.1 we may pick for each $\varphi$ such that $T \nvdash_{\mathcal{G}_{\square \diamond}} \varphi$ a model $M_{\varphi}=\left\langle W_{\varphi}, S_{\varphi}, e_{\varphi}\right\rangle$ such that $e_{\varphi}(w, T)=1$ for all $w$ and $e\left(w_{\varphi}, \varphi\right)<1$. Define $M_{T}=(W, S, e)$ where $W=\amalg_{\varphi} W_{\varphi}, S w w^{\prime}=S_{\varphi} w w^{\prime}$ if $w, w^{\prime} \in W_{\varphi}$ and 0 otherwise, and $e(w, p)=e_{\varphi}(w, p)$ for $w \in W_{\varphi}$. It is easily verified by induction in the complexity of $\theta$ that $e(w, \theta)=e_{\varphi}(w, \theta)$ for any $w \in W_{\varphi}$. Since $M_{T} \models T$ then $T \vdash_{\mathcal{G}_{\square \diamond}} \varphi$ implies $M_{T} \models \varphi$; reciprocally, if $T \nvdash_{\mathcal{G}_{\square \diamond}} \varphi$ then $e\left(w_{\varphi}, \varphi\right)=e_{\varphi}\left(w_{\varphi}, \varphi\right)<1$ by construction and thus $M_{T} \not \models \varphi$.

Theorem 6.2 For any countable bi-modal Gödel algebra $A$ there is Gödel frame $\mathcal{F}_{A}=(W, S)$ such that:

i) $A$ is embeddable in the Gödel complex algebra $[0,1]^{\mathcal{F}_{A}}$.

ii) For any valuation $v: \operatorname{Var} \rightarrow A$ there is a $e_{v}: W \times \operatorname{Var} \rightarrow[0,1]$ such that $v(\varphi)=1$ if and only if $\left(W, S, e_{v}\right) \models \varphi$.

Proof: Fix a valuation $\eta$ into $A$ with onto extension $\eta: \mathcal{L}_{\square \diamond} \rightarrow A$ and let $T=\{\theta: \eta(\theta)=1\}$, then $T$ is normal and for the model $M_{T}=(W, S, e)$ of the previous theorem we have $\eta(\varphi)=1$ if and only if $e(w, \varphi)=1$ for all $w \in W$.

(i) By Theorem 6.1, $e$ induces a bi-modal Gödel valuation $v_{e}:$ Var $\rightarrow$ $[0,1]^{(W, S)}, v_{e}(p)=e(-, p)$ such that $v_{e}(\varphi)=e(-, \varphi)=\mathbf{1} \in[0,1]^{W}$ if an only if $\eta(\varphi)=1$ by the observation above. This means that the 
extension $v_{e}: \mathcal{L}_{\square \diamond} \rightarrow[0,1]^{(W, S)}$ factors injectively through $\eta$; that is, $v_{e}=\delta \circ \eta$ for an injective homomorphism of bi-modal Gödel algebras $\delta: A \rightarrow[0,1]^{(W, S)}$, which shows $(i)$. To see (ii) let $v: \operatorname{Var} \rightarrow A$, then $\delta \circ v$ is a valuation into $[0,1]^{(W, S)}$ which induces, by Theorem 6.1, a GKvaluation $e_{v}: W \times \operatorname{Var} \rightarrow[0,1]$ such that $e_{v}(w, \varphi)=\delta(v(\varphi))(w)$. As $\delta$ is one to one we have that $v(\varphi)=1$ if and only if $\delta(v(\varphi))=\mathbf{1} \in[0,1]^{W}$; that is, $e_{v}(w, \varphi)=1$ for all $w$, which means $\left(W, S, e_{\mu v}\right) \models \varphi$.

Applying part $(i)$ of the previous theorem to the free bi-modal algebra of countable rank we obtain:

Theorem 6.3 The complex algebras generate the variety of bi-modal Gödel algebras. A similar result holds for the subvarieties determined by any combination of identities in (4).

\section{References}

[1] Guram Bezhanishvili and Michael Zakharyaschev, Logics Over MIPC. Proceedings of Sequent Calculus and Kripke Semantics for NonClassical Logics, RIMS Kokyuroku 1021, Kyoto University, 1997, 86-95.

[2] Guram Bezhanishvili, Varieties of Monadic Heyting Algebras. Part I. Studia Logica 61 (1998), 367-402.

[3] Mathias Baaz and Richard Zach, Compact Propositional Logics. Proc. Internationan Symp on multiple valued logic, IEEE Computer Society Press, 1998, pp 108-113.

[4] R.A. Bull, MIPC as formalization of an Intuitionistic concept of modality. JSL 31 (1966) 609-616.

[5] F. Bou, E. Esteva, L. Godo, Modal Systems based on many-valued logics. In New dimensions in fuzzy logic and related technologies. Proceedings EUSFLAT 2007 (Ostrava), volume I, pages 177-182, 2007.

[6] Xavier Caicedo y Ricardo Rodríguez, Standard Gödel Modal Logics. Studia Logic, Volume 94, No. 2 (2010), 189-214

[7] Sergio Celani, Remarks on Intuitionistic Modal Logics. Divulgaciones Matemáticas 9 (2001),137-147. 
[8] A. Chagrov y M. Zakharyaschev, Modal Logic, Clarendon Press, Oxford, 1997.

[9] Davoren, J.M., Topological Semantics and Bisimulations for Intuitionistic Modal Logics and Their Classical Companion Logics In S. Artemov (ed.), Logical Foundations of Computer Science (LFCS 2007), LNCS 4514, pp. 162 - 180, Springer-Verlag, 2007.

[10] Kosta Došen, Models for Stronger Intuitionistic Modal Logics. Studia Logica 44 (1985) 39-70

[11] Pantelis E. Eleftheriou and Costas D. Koutras. Frame constructions, truth invariance and validity preservation in many-valued modal logic. In Journal of Applied Non-Classical Logics. Vol. 15, nro. 4: 367-388. 2005.

[12] Francesc Esteva, Pere Garcia, Lluis Godo, and Ricardo Rodríguez. A modal account of similarity-based reasoning. International Journal of Approximate Reasoning, 16(3-4):235-260, 1997.

[13] G. Fischer Servi, G. The finite model property for MIPQ and some consequences. Notre Dame J.of Formal Logic XIX (1978) 687-692.

[14] G. Fischer Servi. Axiomatizations for some intutitionistic modal logics, Rend. Sem. Mat. Polit de Torino 42 (1984) 179-194

[15] Melving Fitting. Many valued modal logics. Fundamenta Informaticae 15 (1991) 325-254

[16] Melving Fitting. Many valued modal logics, II. Fundamenta Informaticae 17 (1992) 55-73

[17] C. Grefe, Fischer Servis, Intuitionistic modal logic has the finite model property, In Advances in Modal Logic Vol.1 CSLI, Stanford, 1998

[18] P. Hájek, Metamathematics of fuzzy logic, in: Trends in Logic, Vol. 4, Kluwer Academic Publishers, Dordrecht, 1998.

[19] A. Horn, Logic with truth values in a linearly ordered Heyting algebra. JSL 34(3) (1969) 395-409.

[20] C.Koutras, C.Nomikos, P.Peppas, Canonicity and Completeness Results for Many-Valued Modal Logics. Journal of Applied Non-classical Logics $12 / 1$ (2002) 7-42 
[21] P. E. Eleftheriou, C. Koutras, Frame constructions, truth invariance and validity preservation in many-valued modal logic, Journal of Applied Non-classical Logics 15/4 (2005) 367-388

[22] George Metcalfe and Nicola Olivetti: Proof Systems for a Godel Modal Logic. TABLEAUX 2009: 265-279.

[23] A. Monteiro and O. Varsavsky. Algebras de Heyting monádicas. Actas de las X Jornadas de la Unión Matemática Argentina. Bahía Blanca. 1957 , p. $52-62$.

[24] H. Ono. On some intuitionistic modal logics. Publications of the Research Institute for Mathematical Sciences, Kyoto University 13 (1977) $13-55$.

[25] H. Ono \& N.Y.Suzuki, Relations between Intuitionistic modal Logics and Intermediate Predicate Logics. Rep. Math, Logic 22 (1989) 65-87.

[26] Artur Prior. Time and Modality, Clarendon Press, Oxford, 1957.

[27] Laura Rueda, The subvariety of Q-Heyting algebras generated by chains. Revista de la Unión Matemática Argentina 50 (2009) 47-59.

[28] A.K. Simpson. The proof theory and semantics of intuitionistic modal logic. PhD. Thesis, Dept of Computer Science. U. of Edinburgh, 1994.

[29] Frank Wolter, Superintuitionistic Companions of Classical Modal Logics. Studia Logica 58, 3 (1997) 229-295.

[30] F. Wolter and M. Zakharyaschev. Intuitionistic modal logic. In A. cantini, E. Casari, and P. Minari, editors, Logic and Foundations of Mathematics. Kluwer Academic Publishers, 1999, pp. 227-238. 